\title{
Erratum to: Dynamic Traffic Assignment: Theory, Computation and Emerging Paradigms
}

\author{
Terry L. Friesz ${ }^{1} \cdot$ Satish Ukkusuri $^{2}$
}

Published online: 19 September 2015

(C) Springer Science+Business Media New York 2015

\section{Erratum to: Netw Spat Econ \\ DOI 10.1007/s11067-014-9257-3}

The original version of this article unfortunately contained mistakes. Its corrected version is presented below. The title has also been amended as shown above.

Dynamic traffic assignment (DTA) is now an established research specialty overlapping several fields of scholarly enquiry: civil engineering, industrial engineering, operations research, statistics, mathematics, computer science, regional science, city planning, complexity science, sustainability science, and economics. Several hundred scientists and engineers around the globe are devoting substantial energy to DTA research. Moreover, the theory and computational methods arising from DTA research continue to move closer to application and decision support in real-world environments.

In 2012 we issued a call for a broad spectrum of DTA research manuscripts in order to provide a snapshot in time of the diverse points of view being pursued by scholars around the world. Presenters at the Fourth International Symposium on Dynamic Traffic Assignment at Martha's Vineyard, USA, were invited to submit manuscripts for possible publication in Networks and Spatial Economics. From those invitations, we have assembled a collection of 21 papers, each of which has been comprehensively reviewed. Papers 1 through 12 focus on fundamental methodological advances in dynamic traffic assignment modeling and computation, including network loading, continuous time analyses, and day-to-day dynamics. Papers 13 through 21 focus on application of dynamic traffic assignment in the context of transit networks, evacuation modeling, land use modeling, tradable credits, and adaptive control.

The online version of the original article can be found at http://dx.doi.org/10.1007/s11067-014-9257-3.

Terry L. Friesz

tlf13@engr.psu.edu

1 Department of Industrial Engineering, Penn State, University Park, PA 16802, USA

2 School of Civil Engineering, Purdue University, West Lafayette, IN, USA 
The following is a complete list of papers that comprise the Special Issue:

(1) J. Ban: Convergence of Time Discretization Schemes for Continuous-Time Dynamic Network Loading Models

(2) J. Ban: Approximating Time Delays in Solving Continuous-Time Dynamic User Equilibria

(3) S. Boyles: A Continuous DUE Algorithm Using the Link Transmission Model

(4) G. E. Cantarella: Day-to-Day Dynamics \& Equilibrium Stability in a Two-Mode Transport System with Responsive Bus Operator Strategies

(5) M. Carey: Extending the Cell Transmission Model to Multiple Lanes and LaneChanging

(6) X. Di: On the Stability of a Boundedly Rational Day-to-day Dynamics

(7) S. Gao: Combining Disaggregate Route Choice Estimation with Aggregate Calibration of a Dynamic Traffic Assignment Model

(8) E. Ge: A Comparison of Dynamic User Optimal States with Zero, Fixed and Variable Tolerances

(9) T. Iryo: Investigating Factors for Existence of Multiple Equilibria in Dynamic Traffic Network

(10) W. Jin: A New Relationship between Wardrop's User Equilibrium and Nash Equilibrium

(11) Khani: Trip-Based Path Algorithms Using the Transit Network Hierarchy

(12) M. Levin: Improving the Convergence of Simulation-Based Dynamic Traffic Assignment Methodologies

(13) J. Li: Evacuation Planning with Endogenous Transportation Network Degradations: A Stochastic Cell-based Model and Solution Procedure

(14) H. Lo: Combined Route Choice and Adaptive Traffic Control in a Day-to-day Dynamical System

(15) M. Nie: A New Tradable Credit Scheme for the Morning Commute Problem

(16) S. Peeta: Clearance Time Estimation for Incorporating Evacuation Risk in Routing Strategies for Evacuation Operations

(17) G. Ramadurai: A Case for Higher-order Traffic Flow Models in DTA

(18) W. Szeto: A Sustainable Road Network Design Problem with Land Use Transportation Interaction over Time

(19) V. Trozzi: Effects of Countdown Displays in Public Transport Route Choice Under Severe Overcrowding

(20) D. P. Watling: Model Representation \& Decision-Making in an Ever-Changing World: the Role of Stochastic Process Models of Transportation Systems

(21) W. F. Yushimito: Correcting the Market Failure in Work Trips with Work Rescheduling: an Analysis using Bi-level Models for the Firm-workers Interplay 